\title{
PREVALÊNCIA DE QUEDAS EM MULHERES APÓS MENOPAUSA
}

PREVALENCE OF FALLS IN WOMEN AFTER MENOPAUSE

\author{
Débora Aparecida Paccola de Rezende ${ }^{1}$ \\ Wendry Maria Paixão Pereira ${ }^{2}$ \\ Ana Carolina Basso Schmitt ${ }^{3}$ \\ Elaine Cristina Alves Pereira ${ }^{4}$ \\ José Mendes Aldrighi ${ }^{5}$
}

Paccola DA et al. Prevalência de quedas em mulheres após menopausa. Rev. Bras. Cresc. e Desenv. Hum. 2011; 21(1): 146-155.

\section{Resumo:}

Em estudo transversal que tem por objetivo estimar a prevalência de quedas e os fatores associados em mulheres após menopausa até 65 anos. Com amostra randomizada de 331 mulheres, foram aplicados questionários auto-referidos sobre dados de quedas, sócio demográficos, morbidade, medicamentos e hábitos de vida. Foram estimadas a freqüência e análises bivariadas e múltipla com intervalo de confiança de $95 \%$. Os resultados mostraram uma prevalência de quedas nos últimos seis meses da pesquisa de $21,3 \%$, (IC ${ }_{95 \%}: 17.0 \%$ $26.2 \%$ ), das quais $58,8 \%$ sofreram pelo menos uma queda. As quedas ocorreram em ambiente externo e as principais causas foram tropeços, escorregões (49,2\%), tonturas e vertigens (14,9\%). Está associada principalmente com o nível de escolaridade (OR $=2.72$ : $\mathrm{IC}_{95 \%}$ : 1,28 - 5,77), depressão (OR = 2.24; $\mathrm{IC}_{95 \%}: 1,20$ - 4,19) e obesidade $\left(\mathrm{OR}=1.06 ; \mathrm{IC}_{95 \%}: 1,01\right.$ - 1,11). Em conclusão, a prevalência de quedas foi elevada para a faixa etária estudada, evidenciando a necessidade de estratégias de promoção de saúde e prevenção precoce junto à comunidade.

Palavras-chave: epidemiologia; pós-menopausa; acidentes por quedas.

Mestranda do Departamento de Saúde, Ciclos de Vida e Sociedade da Faculdade Saúde Pública/USP.

Doutora pela Faculdade de Saúde Pública/USP.

2,4 Mestres em Saúde Pública da Faculdade de Saúde Pública/USP.

5 Professor Associado do Departamento Materno Infantil da Faculdade de Saúde Pública/USP.

Correspondência para: Débora Aparecida Paccola. Rua: Mateus Grou, 235 - Pinheiros - São Paulo - SP. CEP: 05415-050. E-mail: deb_paccola@yahoo.com.br

Vinculado ao Projeto de Saúde de Pindamonhangaba (PROSAPIN) - Fundação de Amparo a Pesquisa do Estado de São Paulo - FAPESP. Processo nº6/57016-2. 


\begin{abstract}
:
In cross-sectional it was estimated the prevalence of the falls and associated factors in women in postmenopause with age less than 65 years. With a random sample of 331 women, they were applied questionnaire self-reported about falls, socio-demographics, morbidity, medicine and life habits. We estimated the frequency and bivariate and multiple analysis with 95\% confidence interval (95\% CI). The results showed a prevalence of the falls in the last six months of $21.3 \%$ (95\% CI: $17.0-26.2 \%)$, that $58.8 \%$ had at least one fall. The falls occurred in the external environment and the main causes were stumble/slip (48.2\%) and dizziness/vertigo (14.9\%). The fall is associated mainly with the years of schooling $(\mathrm{OR}=2.72$; 95\% CI: 1.28-5.77\%), depression (OR $=2.24$; 95\% CI: 1.65-4.86) and obesity (OR $=1.06 ; 95 \% \mathrm{CI}: 1.01-1.11 \%)$. In conclusion, the prevalence of the falls was high to this population, showing the necessity of strategies of health promotion and early prevention with the community.
\end{abstract}

Key words: epidemiology; postmenopause; accidental falls.

\section{INTRODUÇÃO}

Segundo a Classificação Internacional de Doenças ${ }^{1}$ (CID-10) queda é uma causa externa e após menopausa é intensificada por fatores biológicos e sociais. É também definida como um evento não intencional e que tem como resultado a mudança de posição do indivíduo para um nível mais baixo, em relação a sua posição inicial, acrescido ainda de uma incapacidade de correção em tempo hábil, determinado por circunstâncias multifatoriais que comprometem a estabilidade ${ }^{2,3}$.

Apesar das principais causas conhecidas de quedas serem relatadas em idosos, decorrentes de muitos fatores como alterações na marcha, diminuição da força muscular, deficiência no controle postural, déficit de visão/ audição e uso de medicamentos, também se constata que vários deles também estão presentes em outras faixas etárias ${ }^{4,5}$.

Com o aumento da expectativa de vida, espera-se que a prevalência de quedas aumente ainda mais; por isso sua prevenção assume especial atenção, não só pelas conseqüências que acarretam sobre a qualidade de vida, mas também pelo impacto social e econômico, prin- cipalmente em termos de custo em saúde pública $^{5,6}$.

Além do incremento da expectativa de vida, há outros fatores biológicos no determinismo das quedas. Nesse sentido, merece destaque o climatério, fase da vida da mulher que se caracteriza por progressiva redução na produção de hormônios, especialmente o estrogênio ovariano. O climatério tem início em torno dos 40 anos de idade e pode cursar com sintomas desconfortáveis, como alterações menstruais, calores, secura vaginal, além de sérios agravos como a doença cardiovascular e osteoporose. Durante o climatério, instala-se a última menstruação da mulher - a menopausa - e, em São Paulo ela ocorre em torno de 48,6 anos, sendo que o período da vida posterior a ela é denominado de após menopausa ${ }^{7,8}$.

Além do processo natural do envelhecimento, da falência ovariana, as mulheres após menopausa ingerem somente um terço das necessidades diárias de cálcio, condições que associadamente redundam num maior risco fraturas $^{9}$.

Os estudos sobre quedas são bem conhecidos na população idosa e acarretam déficits financeiros. Entretanto, poucos estudos são 
formulados para populações mais jovens, necessitando investigações de possíveis fatores que levam ao evento de quedas.

Assim, estudar a prevalência de quedas torna-se tema de grande relevância em termos de educação em saúde. Desta maneira, objetiva-se estimar a prevalência de quedas e os fatores associados em mulheres após menopausa até 65 anos.

\section{MÉTODO}

Em estudo observacional vinculado ao Projeto de Saúde de Pindamonhangaba (PROSAPIN), realizado no período de outubro de 2007 a janeiro de 2008, foram avaliadas aleatoriamente 331 mulheres após menopausa, cadastradas no Programa de Saúde da Família, amostra baseada na prevalência de $26 \%$ de quedas ${ }^{10}$, com IC de $95 \%$ e margem de erro de $5 \%$.

Todas participantes foram contatadas em suas casas pelos agentes comunitários de saúde que agendaram entrevista e avaliação antropométrica.

A entrevista foi realizada com um inventário de quedas ${ }^{11}$, composto por 12 questões auto-referidas, incluindo a ocorrência de quedas nos últimos seis meses, uso de dispositivos para auxílio à marcha, local da última queda, às suas circunstâncias, à causa, como caiu, à necessidade de ajuda para se levantar após a queda, à restrição de suas atividades de vida diária, à quase queda e ao medo de cair. Além disso, foram registrados o histórico de fratura, pessoal e familiar (pai e mãe) e a região do corpo fraturada (punho, coluna vertebral e fêmur) ${ }^{12}$.

Outras variáveis foram coletadas, como informações pessoais, dados sócio-demográficos, história ginecológica, morbidades pessoal, questionário de Índice de Qualidade do Sono de Pitsburgh, validado para o português por Ceolin (1999), depressão $0^{14}$, índice de massa corporal, peso e altura foram mensurados de acordo com os critérios da Organização Mundial de Saúde ${ }^{15}$, hábitos de vida como atividade física habitual (IPAC- International Physical Activity Questionnaire) ${ }^{16}$, hábito de fumar ${ }^{17} \mathrm{e}$ uso de medicamentos contínuos ${ }^{18}$.

Todos os questionários foram conferidos no mesmo dia da coleta e checados por pessoas diferentes para maior fidedignidade dos resultados. Foram sorteadas 10\% das participantes para confirmação das repostas visando evitar as imprecisões e os erros sistemáticos.

As frequências de quedas, assim como as demais variáveis de associação referentes à queda foram calculadas e estimadas em análise bivariada e de regressão (ODDS RATIO) com intervalo de confiança 95\% e nível descritivo (valor de pd" 0,05), segundo programa STATA versão 9.1 .

O Comitê de Ética Faculdade Saúde Publica da Universidade de São Paulo aprovou o estudo - protocolo $\mathrm{n}^{\circ} 1776$. O Termo de Consentimento Livre e Esclarecido foi assinado, sendo respeitadas as questões éticas de sigilo e identificação das participantes.

\section{RESULTADOS}

Entre as mulheres após menopausa ( $\mathrm{n}=331$ ) foi observada média de idade de 53,9 anos $(\mathrm{DP}=21,2)$. Predominaram as mulheres casadas (70,2\%), brancas (51,8\%), com menos de quatro anos de estudo (53,5\%) e renda familiar referida pela maioria foi de um salário mínimo, descritas na Tabela 1.

Entre as 331 mulheres após menopausa, 21,3\% (IC 95\%: 17.0\% - 26.2\%) sofreram quedas nos últimos seis meses antes da entrevista, das quais 31 mulheres (59,6\%) sofreram pelo menos uma queda no período. A média de idade das mulheres que caíram foi de 53,5 anos (DP 6,31).

As quedas ocorreram principalmente em ambiente externo $(57,8 \%)$ e a reação automá- 
Tabela 1: Distribuição de frequência das mulheres após menopausa do Programa de Saúde da Família de Pindamonhangaba, 2008.

\begin{tabular}{lrc}
\hline CARACTERÍSTICAS SÓCIO-DEMOGRÁFICAS & $\mathbf{n}$ & $\mathbf{\%}$ \\
\hline Cor & & \\
Branca & 171 & 51,8 \\
Preta & 22 & 6,6 \\
Parda & 132 & 40,0 \\
\hline Religião & 216 & 65,2 \\
Católica & 95 & 28,7 \\
Evangélicas & 20 & 6,0 \\
Demais & & \\
Estado Civil & 224 & 70,2 \\
Casada & 50 & 15,7 \\
Separada/Divorciadas & 45 & 14,1 \\
Viúva & & \\
\hline Escolaridade (em anos) & 161 & 53,5 \\
1 a 4 & 62 & 20,6 \\
5 a 8 & 78 & 25,9 \\
Outros & & \\
\hline Atividade Remunerada & 167 & 50,9 \\
Sim & 161 & 49,1 \\
Não & & \\
\hline Renda Domiciliar (SM*) & 91 & 56,9 \\
Até 1 SM & 69 & 43,1 \\
Mais que 1 SM & & \\
\hline N de Moradores por Domicílio & 22 & 6,7 \\
Moram sozinhas & 147 & 44,8 \\
2 a 3 & 159 & 48,5 \\
4 e mais & & \\
\hline Ocupação & 161 & 48,9 \\
Não trabalham & 47 & 27,6 \\
Serviços domésticos & 94 & \\
Aposentadas/Pensionistas & & \\
Outros & & \\
\hline SM vigene & & \\
\hline & & \\
\hline
\end{tabular}

* SM vigente em 2007 - R\$ 380,00

tica para proteger-se no momento da queda foi referida em $60,9 \%$ das mulheres que caíram, conforme observado na Tabela 2.

Fraturas em punho e coluna devido à queda foram relatadas em $15,5 \%$ das mulheres e histórico familiar de fratura em 15,2\%. Quanto à densidade mineral óssea, apenas 50 mulheres realizaram o exame, no qual osteopenia foi referida por $28,9 \%$ e osteoporose por $24,4 \%$.

A menopausa nesse grupo teve início por volta dos 47 anos $(52,4 \%)$ e somente $18 \%$ fizeram uso de terapia hormonal. Em relação à morbidade, as mulheres relataram ter diabetes (16,1\%) e $15,1 \%$ tiveram algum problema cardíaco. A má qualidade do sono foi considerada por $48,9 \%$, ansiedade mínima por $50,7 \%$ e depressão severa por $6,65 \%$ das entrevistadas. Das 226 mulheres que fazem uso de medicamentos, $62,5 \%$ tomam de 1 a 4 medicamentos contínuos.

Foi calculado o índice de massa corporal e classificadas em sobrepeso, mulheres com IMC de 25 a 29,9 (34,7\%) e obesas, IMC igual ou acima de $30,37,2 \%$. 
Tabela 2: Distribuição de ocorrências de quedas nos últimos seis meses entre as mulheres após menopausa do PSF de Pindamonhangaba, 2008.

\begin{tabular}{lrc}
\hline Características das quedas & $\mathbf{n}$ & $\mathbf{\%}$ \\
\hline Quantidade de quedas & & \\
1 queda & 31 & 59,6 \\
2 a 4 quedas & 16 & 30,7 \\
5 quedas ou mais & 5 & 9,6 \\
\hline Local que ocorreram & 30 & 41,2 \\
Ambiente Doméstico & 41 & 57,8 \\
Ambiente Externo & & \\
Circunstância & 24 & 33,8 \\
Durante uma transferência & 35 & 49,3 \\
Durante a marcha & 12 & 16,9 \\
Outras & & \\
\hline Causas & 33 & 49,2 \\
Tropeços/Escorregões & 13 & 19,4 \\
Obstáculo/Diminuição de atenção & 10 & 14,9 \\
Tontura/Vertigens & 11 & 16,4 \\
Outras & & \\
\hline Mecanismo & 35 & 51,5 \\
Anterior (p/ frente) & 21 & 30,9 \\
Posterior (p/trás) & 12 & 17,6 \\
Lateral (D/E) & & \\
Reação de proteção & 42 & 60,9 \\
Sim & 27 & 39,1 \\
\hline Não & & \\
\hline Ajuda para levantar da queda & 40 & 56,3 \\
Sim & 31 & 43,7 \\
\hline Não & & \\
Quase cair & 194 & 59,1 \\
Sim & 134 & 40,9 \\
\hline Não & & \\
Medo de cair & 230 & 29,9 \\
Sim & 98 & \\
Não & & \\
\hline
\end{tabular}

Em relação aos hábitos diários, como tomar sol, 53\% responderam não ter este costume. Ingestão de suplementos como cálcio e vitamina D foram relatados por 15,6 e 9,30\% das mulheres, respectivamente. Hábitos noturnos, 44,3\% acordavam no meio da noite, três a quatro vezes por semana, para ir ao banheiro.

Segundo a atividade física habitual, $66,5 \%$ consideraram-se ativas, $14,5 \%$ muito ativas e somente $6,67 \%$ sedentárias. A maioria das participantes não fumava $(68,6 \%)$ e não consumiam bebidas alcoólicas (81,9\%).
Fatores de risco para quedas são apresentadas na Tabela 3 em análise bivariada. Quando realizamos análise de regressão (Tabela 4) destaca-se a escolaridade, depressão e obesidade como precursoras ao risco maior de acidentes por quedas.

\section{DISCUSSÃO}

A carência de investigações mais detalhadas sobre a etiologia e metodologia aplica- 
Tabela 3: Descrição das variáveis associadas a quedas referidas, em análise bivariada, odds ratio $(O R$ ), intervalo de confiança (IC) 95\% e nível descritivo (valor de p) das mulheres do Programa de Saúde da Família (PSF) de Pindamonhangaba, 2008.

\begin{tabular}{|c|c|c|c|}
\hline Variáveis & OR & IC (95\%) & $\mathbf{p}$ \\
\hline \multicolumn{4}{|l|}{ Idade (anos) } \\
\hline 50 a 65 & 1,13 & $0,59-2,16$ & 0,692 \\
\hline \multicolumn{4}{|l|}{$\overline{\text { Estado Civil }}$} \\
\hline Casada & 1 & & \\
\hline Separada & 0,59 & $0,31-1,11$ & 0,106 \\
\hline \multicolumn{4}{|l|}{$\overline{\text { Religião }}$} \\
\hline Católica & 1 & & \\
\hline Evangélica & 2,04 & $1,19-3,49$ & $0,009 *$ \\
\hline \multicolumn{4}{|l|}{$\overline{\text { Escolaridade }}$} \\
\hline $1^{\circ}$ colegial e mais & 1,98 & $1,04-3,79$ & 0,037 \\
\hline \multicolumn{4}{|l|}{$\overline{N^{\circ} \text { Moradores }}$} \\
\hline Mais que uma pessoa & 1,00 & $0,89-1,12$ & 0,993 \\
\hline \multicolumn{4}{|l|}{ Depressão } \\
\hline Normal/Leve & 1 & & \\
\hline Média e Severa & 2,89 & $1,68-4,96$ & $0,000 *$ \\
\hline \multicolumn{4}{|l|}{ Qualidade do sono } \\
\hline Ruim & 2,11 & $1,23-3,64$ & $0,007 *$ \\
\hline Obesidade (IMC*) & 1,06 & $1,01-1,10$ & $0,007 *$ \\
\hline \multicolumn{4}{|l|}{ Medo de Cair } \\
\hline Não & 0,56 & $0,30-1,06$ & 0,078 \\
\hline
\end{tabular}

** IMC - Índice de Massa Corporal

* Significativo para $\mathrm{p} \leq 0,05$

da na investigação de quedas, não só entre os idosos, mas também em outras faixas etárias devem ser mais estimuladas, visando uma redução na sobrecarga de atendimento nos serviços de saúde e principalmente dos custos financeiros $^{10,19}$

Este estudo, ao focar uma população antes da velhice, média etária de 53,5 anos bemA presente investigação mostrou essa alta prevalência de quedas, mesmo em mulheres com escolaridade (OR 2,72; IC 1,28 - 5,77), que aliada ao fato de muitas delas morarem sozinhas (OR 1,01; IC 0,88-1,16) puderam justificar o risco de quedas. $\mathrm{O}$ estado civil dessas mulheres não influenciou as associações com as quedas referidas (OR 0,59; IC 0,31-1,11).

Esses dados concordam em parte com outros trabalhos que ao relatarem maior perda de habilidades funcionais nos idosos viúvos e naqueles que moram sozinhos, justificam o alto risco de quedas nessas populações ${ }^{10,11}$, cuja necessidade diária de tarefas domésticas como limpeza e alimentação estão ainda mais prejudicadas por algum tipo de doença crônica, além do que têm menor acesso as informações e serviços de saúde. 
Tabela 4: Análise multivariada entre variáveis associadas a quedas referidas, odds ratio (OR), intervalo de confiança (IC) 95\% e nível descritivo (valor de p) das mulheres do Programa de Saúde da Família (PSF) de Pindamonhangaba, 2008.

\begin{tabular}{lccc}
\hline Variáveis & OR & IC (95\%) & p \\
\hline $\begin{array}{l}\text { Escolaridade } \\
\geq 1^{\circ} \text { colegial }\end{array}$ & 2,72 & $1,28-5,77$ & $0,009^{*}$ \\
\hline $\begin{array}{l}\mathbf{N}^{\mathbf{0}} \text { de Moradores } \\
\text { Morar sozinho }\end{array}$ & 1,01 & $0,88-1,16$ & 0,798 \\
\hline $\begin{array}{l}\text { Idade (anos) } \\
50-65\end{array}$ & 1,27 & $0,61-2,64$ & 0,519 \\
\hline Religião & & & \\
Evangélica & 1,61 & $0,89-3,01$ & 0,134 \\
\hline Morbidades & & & \\
Obesidade & 1,06 & $1,01-1,11$ & $0,011^{*}$ \\
Depressão & 2,24 & $1,20-4,19$ & $0,011^{*}$ \\
Sono Ruim & 1,69 & $0,89-3,10$ & 0,105 \\
\hline Medo de Cair & 0,60 & $0,30-1,21$ & 0,159 \\
\hline
\end{tabular}

* Significativo para $\mathrm{p} \leq 0,05$

Fried e colaboradores (2001) expõem a fragilidade feminina como exemplo de incidência de quedas em faixa etárias maiores; tal fato pode decorrer da redução da massa óssea que se instala em torno de 40 anos $^{24}$, aliada à fraqueza muscular e diminuição da amplitude de movimento em membros inferiores, capazes de provocarem tropeços e desequilíbrio ${ }^{25}$.

O declínio da massa óssea é de $0,5 \%$ por ano, sendo que esta perda se acentua ainda mais (3\% ao ano) após menopausa, devido à falência de produção estrogênica pelos ovários, principal hormônio envolvido na regulação do metabolismo ósseo ${ }^{26}$.

Na presente pesquisa, as mulheres apresentaram fraturas com menor grau de comprometimento (punho e coluna 15,5\%), corroborando com estudo de Siqueira, 2007 que apresentou $12 \%$ de prevalência com algum tipo de fratura. Diferentemente das fraturas por quedas recorrentes, cujas conseqüências são sérias, como limitações da deambulação, mobilidade, podendo resultar em incapacidades permanentes e até a óbito ${ }^{22,25}$.
Uma questão que muito preocupa os profissionais que estudam quedas em mulheres versa sobre o uso de fármacos. Nossos resultados desvelaram o uso médio de 1,6 medicamentos por mulheres, com destaque para os antihipertensivos $(41,4 \%)$, concordando com os resultados de Rozenfeld (2003) e Guimarães (2005) que notaram ainda que as morbidades associadas ao risco de quedas ocorreram tanto na comunidade como em hospitais. Nesse sentido, a Sociedade Americana de Geriatria recomenda diminuição da prescrição de medicamentos, principalmente aqueles que contribuem para o risco, em função de seus efeitos colaterais adversos, como bradicardia, hipotensão, relaxamento muscular e sonolência ${ }^{28}$.

Um resultado muito interessante observado foram morbidades como a depressão e obesidade que representaram os principais fatores associados ao risco de quedas; uma possível justificativa para esse achado reside na possibilidade de estarem inclusas patologias orgânicas, medicações utilizadas, incapacidade funcional, doenças cardíacas ${ }^{29}$. 
Outros fatores incluem o isolamento e dificuldades interpessoais, conflitos com a família, dificuldades econômicas e outros fatores estressantes da vida diária ${ }^{30}$, processo de aposentadoria, morte de companheiro(a) e de amigos.

Assim, é fundamental que os trabalhadores de saúde assumam o compromisso de oferecer à população em processo de envelhecimento uma atenção à saúde que priorize aspectos da promoção de um envelhecimento ativo e capacidade funcional, ao mesmo tempo em que forneça meios para prevenir o desenvolvimento da depressão ${ }^{31}$.

Linhares et al (2003) relatam que a depressão é uma das principais morbidades relacionada a quedas, muitas vezes em decorrência da perda de energia, fraqueza e por conseqüência dificuldades na marcha propiciando as quedas. Autores ${ }^{33}$ apontaram a depressão como fator de risco para quedas devido à desmotivação, isolamento ocasionando a letargia e desatenção nas atividades diárias, ocasionando as quedas.

Em relação à condição física das mulheres, a obesidade subestima não só fator de risco para quedas acidentais, mas risco há um atributo de vida que leva ao sedentarismo, redundando em maior fraqueza muscular (Siqueira e col. 2007). Em nossos resultados, em relação à atividade física mostraram índices de mulheres muito ativas (14,5\%) e somente 6,67\% sedentárias, que também difere de outros estudos onde alterações fisiológicas da

\section{REFERÊNCIAS}

1. Organização Mundial da Saúde. CID-10. São Paulo; 2000. p.1017-9.

2. Moura RN, Santos FC dos, Driemeier M, Santos LM dos, Ramos LR. Quedas em idosos: fatores de risco associados. Gerontologia. 1999;7(2):15-21.

3. Studensk S, Wolter L. Instabilidade e Quedas. In: Duthie, EH, Katz PR. Geriatria idade como desequilíbrio, instabilidades posturais e redução da força muscular são precursoras de quedas (Talbot e col. 2005). Sugestionamos que, em pessoas mais ativas as quedas são atenuadas por forças de maior intensidade no momento do impacto, diferentemente de menos ativas (ou sedentárias), ou mais idosas, onde os riscos de danos são maiores.

Como conclusão, o estudo revelou número expressivo de quedas ainda numa população que não adentrou a terceira idade e que poucos estudos foram conduzidos nessa linha, daí a necessidade de outras investigações nesta faixa etária, para alertar os profissionais de saúde no tocante à prevenção de quedas, desde a adolescência para uma alimentação saudável com cálcio, prática de exercícios visando ganho de massa óssea, além de estimular as autoridades com programas educativos e elaboração de uma política direcionada não só para uma melhor qualidade de vida desse grupo populacional, mas também para minimizar os gastos públicos com internações e custos adicionais por incapacidade.

\section{AGRADECIMENTOS}

À FAPESP e a Secretaria de Saúde do Município de Pindamonhangaba - SP pelo incentivo à pesquisa. À Prof. ${ }^{a}$ Cássia Maria Buchalla por contribuir com o desfecho final deste artigo.

Prática. $3^{\text {a }}$ ed. Rio de Janeiro: Revinter; 2002. p.193-200.

4. Rubenstein CMP, Powers CM, Maclean $\mathrm{CH}$. Quality Indicators for the Management and Prevention of Falls and Mobility Problems in Vulnerable Elders. Ann Intern Med. 2001;135:686-93.

5. Tinetti, Spuchkey M. Prevention of falls among the elderly. The New England journal of medicine. 1989;320: 1055-9. 
6. Brasil. Instituto Brasileiro de Geografia e Estatística, IBGE Censo demográfico: 2005. Disponível em: <http: // www.ibge.gov.br/(2008 ago ).

7. Aldrighi JM, Aldrighi CMS, Aldrighi APS. Alterações sistêmicas no climatério. Rev Bras Med. 2002;59:15-21.

8. Gracia CR, Sammel MD, Freeman EW, Lin H, Langan E, Kapoor S, et al. Defining menopause status: creation of a new definition to identify the changes of the menopause transition. Menopause. 2005;12(2):128-135.

9. Evci ED, Ergin F, Beser E. Home accidents in the elderly in Turkey. Tohoku Exp Med. 2006;209(4):291-301.

10. Lebrão ML, Laurenti R. Saúde, bem-estar e envelhecimento: o estudo SABE no município de São Paulo. Rev Bras Epidemio. 2005;8(2):127-41.

11. Perracini MC, Ramos R. Fatores associados a quedas em uma coorte de idosos residentes na comunidade. Rev de Saúde Pública. 2002;36(6):709-16.

12. Dontas IA, Yiannakopoulos CK. Risk factors and prevention of osteoporosis related fractures. J Musc Neuronal Interact. 2007;jul-set;7(3):268-72.

13. Ceolin MF. Padrões de atividade e fragmentação do sono em pessoas idosas. São Paulo (SP), 242 p. (tese de doutorado). Escola de Enfermagem da USP; 1999.

14. Gorenstein C, Andrade L. Validation of a Portuguese version of the Beck Depression Inventory and the State-Trait Anxiety Inventory in Brazilian Subjects. Brazilian Journal of Medical and Biological Researchp.1996; p.453-457.

15. WHO World Health Organization. Obesity: preventing and managing the global epidemic (report of a WHO consultation on obesity). Genebra: World Health Organization. 1997; p.98.

16. Matsudo SM, Matsudo VR, Araújo T, Andrade D, Andrade E, Oliveira L, et al.
Nível de atividade física da população do Estado de São Paulo: análise de acordo com o gênero, idade, nível socioeconômico, distribuição geográfica e de conhecimento. Rev Bras Ciên e Mov. 2002; v.10, n.4, p.41-50.

17. Alves HNP, Surjan JC, Nogueira-Martins LA, Marques ACPR, Ramos SP, Laranjeira RR. Perfil Clínico e Demográfico de Médicos com Dependência Química. Rev Assoc Med Bras. 2005; 51(3):139-43.

18. ANVISA. Agência de Vigilância Sanitária. Disponível em: http://www.anvisa.gov.br/ medicamentos/index.htm (2008 out).

19. Perell KL, Nelson A, Goldman RL, Luther SL, Prieto-Lewis N, Rubenstein LZ. Fall Risk Assessment Measures: An Analytic Review. Journal of Gerotology. 2001; v.56, n.12, p.761-766.

20. Rubenstein LZ. Falls in older people: epidemiology, risk factors and strategies for prevention. Age Ageing. 2006; 35(Suppl 2):ii37-41.

21. Rozenfeld S, Camacho LAB, Veras RP. Medication as a risk factor forfalls in older women in Brazil. Rev Panam Salud Publica/Pan; Am J Public Health. 2003;13(6).

22. Fabrício SCC, Rodrigues RAP, Costa Junior ML. Causas e conseqüências de quedas de idosos atendidos em hospital público. Rev Saúde Pública. 2004; 38(1):93-9.

23. Fried LP, Tangen CM, Walston J, Newman AB, Hirsh C, Gottdiener J, et al. Frailty in older adults. J Gerontol A Biol Sci Med Sci. 2001; v.56, p.146-57.

24. National Osteoporosis Foundation. Osteoporosis Report. 1999; 15, n.4.

25. Francis RM. Falls and fractures. Age and Ageing. Br Geriatrics Soc. 2001; 30-54; 25-28.

26. Matsudo VK, Matsudo SM, Andrade DR, Araújo TL, Oliveira LC, Bragion GF. Promotion of physical activity in a developing country: The Agita São Paulo 
experience. Public Health Nutr. 2002;5(1a):1-10.

27. Guimarães JMN, Farinatti PTV. Análise descritiva de variáveis teoricamente associadas ao risco de quedas em mulheres idosas. Revista Bras Med Esporte. 2005; v.11, n.5, set/out.

28. American Geriatrics Society; British Geriatrics Society \& American Academy of Orthopaedic Surgeons Panel on Falls Prevention. Guidelines for the prevention of falls in older persons. J Am Geriatr Soc. 2001;49:664-72.

29. Toledo MAV, Santos Neto LS. Depressão no idoso In: Hargreaves LH, organizador. Geriatria. Brasília: Prodasen; 2006. p.545-52.

30. Brasil Ministério da Saúde. Envelhecimento e saúde da pessoa idosa. Cadernos de Atenção Básica, n. ${ }^{\circ} 19,2006$.

31. Coutinho ES, Silva SD. Uso de medicamentos como fator de risco para fratura grave decorrente de queda em idosos. Cad Saúde Pub. 2002;18:1359-66.
32. Linhares CRC, Guimarães RM, Campos APM, Carvalho NTC, Decnop VL. Perfil da clientela de um ambulatório de geriatria do Distrito Federal. Psicologia Reflexão e Crítica, 2003;16(2):19-326.

33. Canavillas B A, Padilla RF, Jiménez MJJ, Peinado ACA, Gálvez VR. Risk factors in falls among the elderly according to extrinsic and intrinsic precipitating causes. European Journal of Epidemiology. 2001;16:849-59.

34. Siqueira FV, Facchini LA, Piccini RX, Tomasi E, Thumé E, Silveira DS, et al. Prevalência de quedas em idosos e fatores associados. Rev de Saúde Pública. 2007; 41(5):749-56.

35. Talbot LA, Musiol RJ, Witham EK, Metter EJ.Falls in young, middle-aged and older community dwelling adults: perceived cause, environmental factors and injury. BMC Public Health. 2005; Aug 18;5-86.

Recebido em: 06/out./10

Modificado em 28/out./10

Aprovado em 28/dez./10 